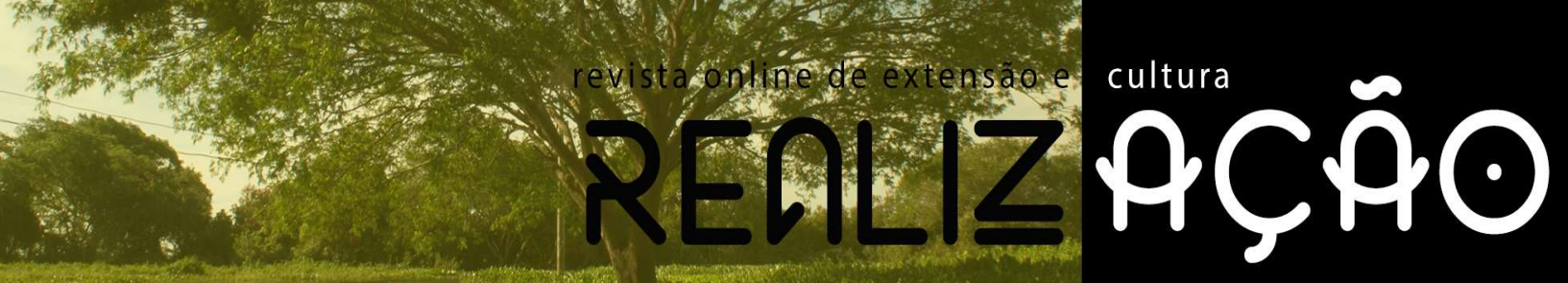

\title{
Ações de eextensão da UNIMONTES na difusão de tecnologias zootécnicas à agricultores da Região Semiárida do Norte de Minas Gerais
}

\section{Extension actions of Unimontes in diffusion of zootechnics technologies to farmers in the north Minas Gerais Region}

DOI

10.30612/re-ufgd.v6i12.10643

\author{
Flávio Pinto Monção ${ }^{1}$ \\ Vicente Ribeiro Rocha Júnior ${ }^{2}$ \\ Osmar Antunes Neto ${ }^{3}$ \\ Leidy Darmony de Almeida Rufino ${ }^{4}$ \\ Cinara da Cunha Siqueira Carvalho ${ }^{2}$ \\ Amanda Cristian Cardoso Prudêncio ${ }^{2}$ \\ Maria Luiza Oliveira ${ }^{2}$ \\ Raissa Carvalho Nascimento ${ }^{2}$ \\ Dijair Barbosa Leal ${ }^{2}$ \\ Heberth Christian Ferreira²
}

Recebido em 06/11/2019 - Aceito em 16/12/2019

Resumo - Objetivou-se por meio das atividades extensionistas orientar e incentivar produtores da agricultura familiar da região semiárida do Norte de Minas Gerais quanto ao uso de tecnologias de forma estratégica visando aumentar a produção de leite e/ou carne ao longo do ano. $\mathrm{O}$ desenvolvimento das ações para os produtores familiares ocorreram nas cidades de Espinosa, Catuti, Porteirinha, Janaúba e Verdelândia a partir de Julho de 2017. Foram realizadas reuniões com a Secretaria de Agricultura do município e com as famílias produtoras, com a finalidade de interagir e explicar o papel da Unimontes no local, bem como a troca de saberes e conhecimento histórico, área local e a comunidade como um todo. Houve participação de 514 produtores rurais nas palestras discutindo manejo e uso da palma forrageira, BRS capiaçu e estratégias de suplementação para ruminantes. Houve participação de 214 produtores nas reuniões realizadas em Espinosa e 70 produtores na zona rural de Catuti. Detecta-se a importância da continuidade das palestras e orientações técnicas aos produtores da região semiárida com intuito de incrementar a produção animal/vegetal, a oferta de produtos de melhor qualidade e a fonte de renda dos envolvidos no sistema de produção.

Palavras-chaves: Catuti, Espinosa, Janaúba, Produtores rurais, orientação.

Abstract: The objective of this study was to guide and encourage family farmers in the semi-arid region of northern Minas Gerais to use technologies strategically to increase milk and/or meat production throughout the year. The development of actions for family producers took place in the cities of Espinosa, Catuti, Porteirinha, Janaúba and Verdelândia from July 2017. At first, meetings were held with the municipality's Agriculture Secretariat and with the producing families, with the

\footnotetext{
1 UNESP

2 UNIMONTES

3 EMATER-MG

4 EPAMIG-Norte
} 
purpose of to interact and explain Unimontes' role in the place, as well as the exchange of knowledge and historical knowledge, local area and the community as a whole. 514 farmers participated in the lectures discussing management and use of cactus pear, BRS capiaçu and supplementation strategies for ruminants. 214 producers attended the meetings held in Espinosa and 70 producers in rural Catuti. The importance of the continuity of the lectures and technical orientations to the producers of the semi-arid region is detected in order to increase the animal/vegetal production, the offer of better quality products and the source of income of those involved in the production system.

Keywords: Catuti, Espinosa, Janaúba, Rural producers, Orientation.

\section{Introdução}

No Brasil, a região semiárida ocupa uma área de 928,56 mil km², sendo esta cerca de $11 \%$ do território nacional, abrangendo os sertões do estado da Bahia, Sergipe, Alagoas, Pernambuco, Paraíba, Rio Grande do Norte, Ceará, Piauí, mais 45 municípios do sudeste do Maranhão e uma parte do norte dos estados de Minas Gerais e Espírito Santo (Ferreira et al. 2009).

Estima-se que na região Norte de Minas Gerais, a área caracterizada como semiárida seja de 177 mil km². A Universidade Estadual de Montes Claros (Unimontes), com sede geral na cidade Montes Claros, possui 10 campi distribuídos na região semiárida do Norte de Minas com a finalidade de formar profissionais e, principalmente, desenvolver ciência e tecnologia para o crescimento regional. No que tange as atividades desenvolvidas no meio rural, agronegócio, ainda existem lacunas no conhecimento por parte dos produtores e técnicos quanto ao manejo adequado de alguns sistemas de produção (i.e. leite, carne).

Em função das longas estiagens, os pequenos produtores ficam vulneráveis quanto à certeza da lucratividade das atividades desenvolvidas no meio rural, o que dificulta a contratação de técnicos para orientações técnicas diversas sobre os sistemas de produção específicos. Normalmente, as orientações aos produtores ocorrem em lojas agropecuárias ou órgãos especializados do governo, mas, possivelmente devido à extensão territorial, há uma carência de acompanhamento técnico em nível de campo dos envolvidos com a produção de alimentos.

Nesse sentido, à Unimontes em parceria com a Emater, Epamig e outras universidades, por meio das ações de extensão, tem um papel fundamental na transferência de tecnologias agronômicas e zootécnicas aos agricultores familiares localizado na região semiárida do Norte de Minas, possibilitando a inclusão social de produtores, principalmente os menos favorecidos, ao acesso a informações e orientações técnicas de qualidade. Além disso, as atividades extensionistas no meio rural é uma estratégia de garantir e antecipar a chegada dos conhecimentos 
geradas nos centros acadêmicos por meio de pesquisas aos produtores rurais como relatados nos trabalhos de Oliveira et al., (2012; 2016; 2017), Gabriel et al., (2015), Monção et al. (2019c). Esses autores desenvolveram atividades extensionistas em diversas comunidades da região da Grande Dourados e Sul do Estado de Mato Grosso do Sul desde 2007 e reportaram avanços de desenvolvimentos significativos nas comunidades rurais e, sobretudo, para o município de Dourados. Essas metodologias de extensão rural e universitária propostas nos trabalhos de Oliveira et al., $(2012 ; 2016$; 2017) não são específicas, contudo, podem ser aplicadas e adaptadas em outras regiões como as pertencentes ao Norte de Minas.

Com base no exposto, objetivou-se por meio de atividades extensionistas orientar e incentivar produtores da agricultura familiar em diversos municípios pertencentes à região semiárida do Norte de Minas quanto ao uso de tecnologias de forma estratégica visando à melhoria da produção de leite e/ou carne ao longo do ano.

\section{Material e Métodos}

O desenvolvimento das ações para os produtores familiares da região do semiárido do Norte de Minas Gerais foi realizado a partir de Julho de 2017. Inicialmente, as cidades contempladas com as ações de extensão foram Espinosa, Catuti, Janaúba, Verdelândia e Porterinha. A princípio, foram realizadas reuniões com a secretaria de Agricultura e Emater de cada município e com as famílias com a finalidade de interagir e explicar o papel da Unimontes no local, bem como a troca de saberes e conhecer o histórico, área local e a cada sub-região como um todo. Posteriormente, foram realizadas reuniões participativas para planejamento de implantação e desenvolvimento de atividades, onde foram formados grupos mediante as atividades passíveis de serem implantadas.

Palestras de cunho social e técnicas e cursos teórico-práticos foram desenvolvidas com o intuito de despertar o interesse das comunidades às novas possibilidades de produção e aprimorar as existentes. Durante as reuniões foram utilizados data show, banners e folders, dentre outros recursos para transmitir a informação aos produtores. Todo material didático e de uso no campo, como sementes, entre outros, foram adquiridos através de lojas locais e auxílios de programas/projetos desenvolvidos pela Unimontes/Janaúba e instituições parceiras como as secretarias de agricultura e pecuária juntamente com a EMATER e Epamig regional e local.

Dentre as atividades realizadas destacam-se as palestras oferecidas para todas as comunidades de cada município envolvidas e oficinas realizadas estrategicamente. Foram 
abordados assuntos sobre importância da mineralização e correção dos nutrientes das dietas dos animais durante o ano todo. Além disso, foi abordada a relevância do balanceamento da dieta dos animais, sobretudo os com aptidão leiteira. Alguns produtores que não puderam, de alguma forma, participar das reuniões mensais no auditório da EMATER e Sindicato Rural foram orientados em suas propriedades quanto ao uso das tecnologias e sanadas diversas dúvidas na área de produção animal/vegetal.

Foram utilizados folders e cartilhas sobre o manejo dos suplementos, assim como a importância da adaptação dos animais, como escolher o suplemento correto em função da análise do pasto. Os produtores também foram orientados em como fazer misturas múltiplas (suplementos concentrados) balanceadas na propriedade visando reduzir os custos com à alimentação.

Outro tema bastante discutido por meio de palestras e oficinas foi suplementação volumosa dos animais por meio na implantação, cultivo e uso de palma forrageira e BRS capiaçu (Pennisetum purpureum Schum.). Foram utilizados folders e cartilhas sobre o manejo da palma e BRS capiaçu, incluindo a escolha do local para plantio, preparo do solo, escolha das mudas, plantio, adubação, colheita e uso em dietas de ruminantes. A prefeitura local (Espinosa), por meio da secretaria de agricultura e pecuária, forneceu suporte para implantação de uma unidade demonstrativa de palma forrageira e capineiras em uma das comunidades como forma de oferecer mudas aos produtores envolvidos. Nas outras cidades, as mudas doadas foram oriundas da Fazenda Experimental da Unimontes/Janaúba.

As palestras e reuniões foram divulgadas por meio de contato verbal dos lojistas da região, cartazes e por distribuição de folders em locais estratégicos pela secretaria de agricultura e pecuária municipal e Emater.

\section{Resultados e Discussão}

Inicialmente, em todos os municípios, alguns produtores resistiram em participar das reuniões e palestras, principalmente na cidade de Espinosa, talvez por não acreditar nesse elo de extensão entre a Universidade e a Comunidade. Além disso, devido ao horário das reuniões, muitos produtores envolvidos com à atividade leiteira não puderam participar das palestras. Contudo, houve participação de 40 produtores de várias comunidades no primeiro encontro de extensão desenvolvido no auditório da Emater em Espinosa. Na cidade de Espinosa, com 92 comunidades rurais, houve participação de 214 produtores distribuídos em diferentes palestras e locais. Em seguida, os eventos realizados em Porteirinha tiveram participação de 200 produtores (Tabela 1). 
Tabela 1. Distribuição das palestras nas diferentes cidades de Minas Gerais e número de participantes

\begin{tabular}{rrrc}
\hline \multicolumn{1}{r}{ Cidade } & Local/Comunidade & $N^{0}$ de reuniões & Total de participantes \\
\hline Espinosa & Juremal & 1 & 6 \\
Espinosa & Espigão & 1 & 28 \\
Espinosa & Auditório & 1 & 40 \\
Espinosa & Auditório & 1 & 60 \\
Espinosa & Auditório & 1 & 80 \\
Catuti & Zona rural & 1 & 70 \\
Porteirinha & Zona rural & 1 & 80 \\
Porteirinha & Sindicato rural & 1 & 120 \\
Janaúba & Parque de Exposição & 1 & 30 \\
\hline
\end{tabular}

Em geral, as ações de extensão da Unimontes atenderam 514 produtores rurais do semiárido e verifica-se a demanda de mais palestras e orientações por parte dos produtores rurais. Na região semiárida do Norte de Minas, a principal limitação dos sistemas de produção de leite e carne consiste na oferta quantitativa de volumosos. Na prática, o capim-bufel (Cenchrus ciliaris) e capim-corrente (Urochloa mosambicensis (Hanck). Dandy) são os mais cultivados, contudo, a massa de forragem conservada para o período da seca somente dessas forrageiras nem sempre é suficiente para a quantidade de animais.

Trabalhar com espécies forrageiras que conseguem produzir na região semiárida, resistente a seca, é o maior interesse e desafio dos produtores. Nesses municípios do semiárido Norte Mineiro, há cinco anos (2012/2017) teve as precipitações distribuída irregularmente, o que potencializou os efeitos do período da seca. Com isso, as ações de extensão com ênfase na produção de palma forrageira (Opuntia e Nopalea) e capim-BRS capiaçu ganharam destaque. A palma forrageira é uma das poucas forrageiras existente no semiárido que consegue produzir em ambientes hostis de umidade, e isso despertou interesse de muitos produtores de leite da região em participar das orientações técnicas. A palma forrageira, nas condições de sequeiro, produz 12 a 25 t/ha, com média de $60 \%$ de carboidratos não fibrosos e $55 \%$ de nutrientes digestíveis totais (Ferreira et al. 2012). É um alimento rico em energia e água para os animais que estão em produção em regiões cuja disponibilidade de água, em alguns locais, é bastante limitada. O teor de energia da palma é um grande aliado ao produtor na redução dos custos com outros alimentos como Milho usado na dieta dos animais que não é produzido em alta escala nessas regiões. A região semiárida do Norte de Minas apresenta elevado potencial para crescimento quanto se trata da produção de alimentos derivados do leite.

É de conhecimento internacional a qualidade dos queijos produzidos na cidade de 
Porteirinha. Contudo, na região como um todo, a média de produtividade das vacas mestiças Holandês/Zebu, que são responsáveis por mais de $80 \%$ do volume de leite produzido, é abaixo de 1,4 t/animal/lactação. Isso é justificado principalmente pelas dietas desbalanceadas desses animais, o que destaca a importância do cultivo de forrageiras com elevado potencial produtivo de massa como suplementação volumosa para os animais. De acordo com Monção et al., (2019 ab), o BRS capiaçu tem potencial para produção de até 72 toneladas (irrigado no Inverno) de matéria seca por hectare ano na região de Janaúba, Minas Gerais com bom valor nutricional. Atualmente, muitas das palestras consistem na orientação aos produtores quanto a melhor época para corte do BRS capiaçu para produção de silagem ou para corte diário.

No município de Verdelândia, foram doadas um mil mudas de BRS capiaçu e 800 mudas de palma forrageira, variedade IPA Sertânia, para alguns produtores que se disponibilizaram em implantar e manejar unidades demonstrativas. Essas ações de extensão têm melhorado consideravelmente a vida dos produtores rurais nessas cidades, além de melhorar a qualidade dos alimentos produzidos. Isso porque, nos Dias-de-campo, além de abordar o manejo das forrageiras é também ministrada palestras com relação ao manejo sanitário.

\section{Conclusão}

Detecta-se a importância da continuidade das palestras e orientações a técnicas aos produtores da região semiárida do Norte de Minas Gerais com intuito de incrementar a produção animal/vegetal, a oferta de produtos de melhor qualidade e a fonte de renda dos envolvidos.

\section{Agradecimentos}

Os autores agradecem à Unimontes, Fundação de Amparo à Pesquisa do Estado de Minas Gerais (FAPEMIG), ao Banco do Nordeste do Brasil (BNB), Conselho Nacional de Desenvolvimento Científico e Tecnológico (CNPq), EMATER Minas Gerais e EPAMIG pelo suporte financeiro e pelas bolsas de Estudos. Este estudo foi financiado em parte pela Coordenação de Aperfeiçoamento de Pessoal de Nível Superior (CAPES) - Código Financeiro 001.

\section{Referências}

FAEMG- Palmas para Minas. http://www.sistemafaemg.org.br/Noticia.aspx? $\underline{\text { Code }}=13992 \&$ Portal $=1 \&$ PortalNews $=1 \&$ ParentCode $=139 \&$ ParentPath $=$ None \&ContentVersion $=\mathrm{R}$. Acessado em Maio de 2018. 


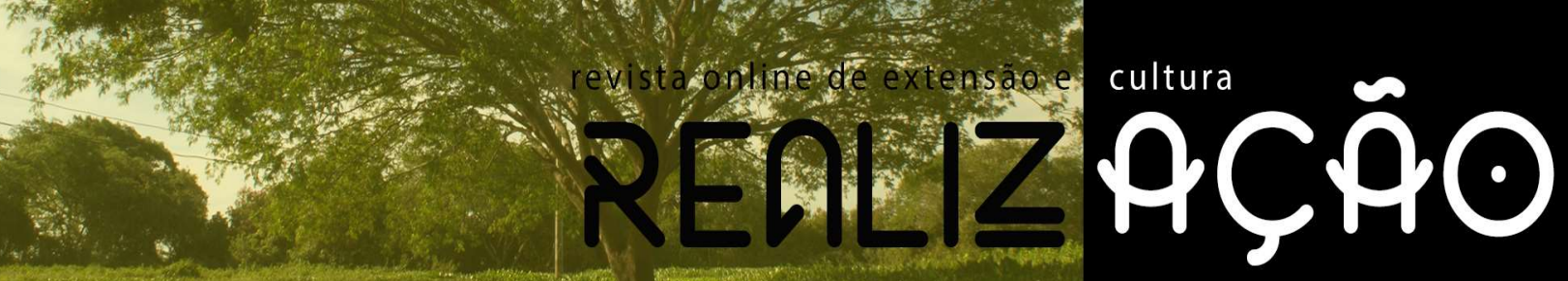

FERREIRA, M.A., BISPO, S.V., ROCHA FILHO, R.R., URBANO, S.A., COSTA, C.T.F. The use of cactus as forage for dairy cows in semi-arid regions of Brazil. In: PetrKon valina.(Org.), Organic Farming and Food Production. InTech, South Bohemia, 2012. p. 1-22.

GABRIEL, A.M.A.; SOUZA, R.; OLIVEIRA, E.R.; ROSSINI, L.C.; MONÇÃO, F.P.; RAMOS, M.B.M.; GIMENES, L.S.; PEREIRA, T.L.; SILVA, E.C.P. Orientação em apiários noAssentamento Amparo, Dourados-MS. Revista online de Extensão e Cultura Realização, v. 2, n.3, p. 36-41, 2015.

MONÇÃO, F.P.; COSTA, M.A.M.S.; RIGUEIRA, J.P.S.; MOURA, M.M.A.; ROCHA JÚNIOR, V.R.; GOMES, V.M.; LEAL, D.B.; MARANHÃO, C.M.A.; ALBUQUERQUE, C.J.B.; CHAMONE, J.M.A. Yield and nutritional value of BRS Capiaçu grass at different regrowth ages. Semina Ciências Agrárias, v.41, n.5, 2019b.

MONÇÃO, F.P.; COSTA, M.A.M.S.; RIGUEIRA, J.P.S.; SALES, E.C.J.; LEAL, D.B.; SILVA, M.F.P.; GOMES, V.M.; CHAMONE, J.M.A.; ALVES, D.D.; CARVALHO, C.C.S.; MURTA, J.E.J.; ROCHA JÚNIOR, V.R. Productivity and nutritional value of BRS capiaçu grass (Pennisetum purpureum) managed at four regrowth ages in a semiarid region. Tropical Animal Health and Production, v.51, p. 1-7, 2019a.

MONÇÃO, F.P.; ALKIMIN, J.M.; RIGUEIRA, J.P.S.; TOLENTINO, D.C.; ROCHA JÚNIOR, V.R.; CHAMONE, J.M.A.; CARVALHO, C.C.S.; MARQUES, O.F.C.; MELO, J.A.R.; SILVA, M.F.P.; SALES, E.C.J.; ROCHA, M.H. Transferência de tecnologias zootécnicas a agricultores familiares no município de Espinosa/MG. Revista online de Extensão e Cultura Realização, v. 6, n. 11, p. $84-139,2019$ c.

OLIVEIRA, E.R.; GABRIEL, A.M.A.; MONÇÃO, F.P.; FARIAS, M.F.L.; PEIXOTO, P.P.P.; RAMOS, M.B.M.; MOURA, L.V.; PEREIRA, T.L.; GANDRA, J.R.; SANTOS JUNIOR, E.A. Extensão universitária como estratégia de desenvolvimento na Comunidade Quilombola de Dourados/MS. Revista online de Extensão e Cultura Realização, v. 3, n.1, p. 35-44, 2016 b.

OLIVEIRA, E.R.; MONÇÃO, F.P.; RAMOS, M.B.M.; GABRIEL, A. M. A.; FARIAS, M.F.L.; MOURA, L.V. Práticas extensionistas no desenvolvimento sustentável da comunidade quilombola de dourados, mato grosso do sul. Em Extensão, v. 11, n. 2, p. 82-95, 2012.

OLIVEIRA, E.R.; MUNIZ, E.B.; GABRIEL, A.M.A.; MONÇÃO, F.P.; GANDRA, J.R.; GANDRA, E.R.S.; PEREIRA, T.L.; SILVA, M.S.J.; GOUVEA, W.S.; CARMO, A.A.; PEDRINI, C.A.; BECKER, R.A.S. Produção de feno orgânico como estratégia de suplementação volumosa para ruminantes produzidos nas comunidades rurais de mato grosso do sul. Revista online de Extensão e Cultura Realização, v.4, n.8, p-87-97, 2017. 